\title{
BMJ Open Incidence, risk factors and prognostic effect of imaging right ventricular involvement in patients with COVID-19: a dose-response analysis protocol for systematic review
}

Chenghui Zhou (D) , ${ }^{1}$ Baohui Lou, ${ }^{2}$ Hui Li, ${ }^{3}$ Xin Wang, ${ }^{4}$ Hushan Ao, ${ }^{1}$ Fujian Duan ${ }^{4}$

To cite: Zhou C, Lou B, Li H, et al. Incidence, risk factors and prognostic effect of imaging right ventricular involvement in patients with COVID-19: a doseresponse analysis protocol for systematic review. BMJ Open 2021;11:e049866. doi:10.1136/ bmjopen-2021-049866

- Prepublication history for this paper is available online. To view these files, please visit the journal online (http://dx.doi org/10.1136/bmjopen-2021049866).

$\mathrm{CZ}$ and $\mathrm{BL}$ are joint first authors

Received 03 February 2021 Accepted 19 April 2021

Check for updates

(C) Author(s) (or their employer(s)) 2021. Re-use permitted under CC BY-NC. No commercial re-use. See rights and permissions. Published by BMJ.

For numbered affiliations see end of article.

Correspondence to

Dr Fujian Duan;

duanfujian@aliyun.com

\section{ABSTRACT}

Introduction Emerging evidence has shown that COVID-19 infection may result in right ventricular (RV) disturbance and be associated with adverse clinical outcomes. The aim of this meta-analysis is to summarise the incidence, risk factors and the prognostic effect of imaging RV involvement in adult patients with COVID-19. Methods A systematical search will be performed in PubMed, EMBase, ISI Knowledge via Web of Science and preprint databases (MedRxiv and BioRxiv) (until October 2021) to identify all cohort studies in adult patients with COVID-19. The primary outcome will be the incidence of RV involvement (dysfunction and/or dilation) assessed by echocardiography, CT or MRI. Secondary outcomes will include the risk factors for RV involvement and their association with all-cause mortality during hospitalisation. Additional outcomes will include the RV global or free wall longitudinal strain (RV-GLS or RV-FWLS), tricuspid annular plane systolic excursion (TAPSE), fractional area change (FAC) and RV diameter. Univariable or multivariable metaregression and subgroup analyses will be performed for the study design and patient characteristics (especially acute or chronic pulmonary embolism and pulmonary hypertension). Sensitivity analyses will be used to assess the robustness of our results by removing each included study at one time to obtain and evaluate the remaining overall estimates of RV involvement incidence and related risk factors, association with all-cause mortality, and other RV parameters (RV-GLS or RV-FWLS, TAPSE, S', FAC and $\mathrm{RV}$ diameter). Both linear and cubic spline regression models will be used to explore the dose-response relationship between different categories $(>2)$ of RV involvement and the risk of mortality (OR or HR).

Ethics and dissemination There was no need for ethics approval for the systematic review protocol according to the Institutional Review Board/Independent Ethics Committee of Fuwai Hospital. This meta-analysis will be disseminated through a peer-reviewed journal for publication.

PROSPERO registration number CRD42021231689.

\section{INTRODUCTION}

By 31 January 2021, the COVID-19 pandemic had caused 103495393 infections and
Strengths and limitations of this study

- A comprehensive linear or cubic dose-response analysis between different categories $(>2)$ of right ventricular involvement and the risk of mortality in patients with COVID-19 will be performed.

- Sensitivity analyses will be performed to assess the robustness of the association of right ventricular involvement with all-cause mortality .

- The effect of different severities of COVID-19 associated with right ventricular involvement on adverse clinical outcomes merits further investigation.

- The study will be retrospective in design and the inherit bias cannot be ruled out.

- The sample size and the number of included studies may be inadequate.

2236883 deaths worldwide in 215 countries. ${ }^{1}$ Since the initial outbreak, the overall mortality risk of patients with COVID-19 has remained stable at approximately $2.0 \%$. However, critically ill patients admitted to the intensive care unit (ICU) continue to be exhibit a high mortality of up to $20 \%-50 \% .^{2-4}$ The major contributing complication is thought to be the cardiovascular involvement, manifesting as biomarker elevation of myocardial injury, ST-segment elevation, decrease in ventricular function and change in ventricular structure. ${ }^{5}$ In a retrospective analysis, Zhou et al showed that the incidence of heart failure after COVID-19 infection is up to $52 \%$ in deceased patients and approximately $12 \%$ in discharged patients. ${ }^{6}$

The right ventricles (RVs) have been recognised as passive conduits and volumetric organs, and are vulnerable to changes in preload and afterload. In clinical settings, therapy for patients with RV failure is less effective than for patients with left ventricular (LV) failure, resulting in poor medium-term 
and long-term outcomes. ${ }^{7-9}$ Various pharmacological interventions have been studied with controversial results. Isolated reports suggest that administration of promising drugs may reduce ICU stays, but their effectiveness regarding mortality remains elusive. ${ }^{10}{ }^{11}$ Hence, early identification of RV involvement could lead to risk stratification, early intervention and potentially improve the prognosis for critically ill patients with COVID-19.

The role of RV in cardiovascular medicine has become a heavily researched issue. In studies concerning COVID-19 related ventricular function, involvement of RV as opposed to LV may be more frequent ${ }^{12}{ }^{13}$ and have more prognostic significance. ${ }^{14}$ Therefore, understanding the incidence and risk factors for imaging RV involvement following COVID-19 infection has critical clinical implications, including more precise targeting of interventions and the development of a sensitive risk screening tool. ${ }^{12-15}$ Additionally, whether different severities of RV involvement have different effects on clinical outcomes remains unknown. ${ }^{16-18}$ However, there has been no metaanalysis concerning this important issue. Therefore, we will conduct a comprehensive meta-analysis to quantitatively evaluate the incidence, risk factors and prognostic effect of imaging RV involvement in patients with COVID-19.

\section{Objectives}

The aim of this systematic review and meta-analysis is to summarise the evidence of incidence, risk factors and prognostic effect of imaging RV involvement in patients with COVID-19.

\section{METHODS AND ANALYSIS}

\section{Search strategy}

This systematic review and meta-analysis will be performed in accordance with the Preferred Reporting Items for Systematic Review and Meta-Analysis Protocols. ${ }^{19}$ PubMed, EMBase, ISI Knowledge via Web of Science and preprint databases (MedRxiv and BioRxiv) (until October 2021) will be systematically searched to identify all cohort studies concerning imaging RV involvement in adult patients with COVID-19. The imaging methods will be echocardiography, cardiac CT and cardiac MRI. The reference lists of the retrieved articles will also be searched. The related search strategy with keywords is presented in table 1 . The searching process is shown in figure 1.

\section{Type of participants}

Adult patients infected with COVID-19 will be selected as the study participants.

\section{Patient and public involvement}

Patients and/or the public will not be involved in the design, conduct or reporting or dissemination plans for this research.

\section{Type of studies}

We will include both retrospective and prospective cohort studies concerning imaging RV in adult patients with
Table 1 Search strategy in various databases (PubMed, EMBase, ISI Knowledge via Web of Science, MedRxiv and BioRxiv)

\begin{tabular}{|c|c|}
\hline Database & Search items \\
\hline \multicolumn{2}{|l|}{ PubMed } \\
\hline \multicolumn{2}{|l|}{ No. } \\
\hline \# 1 & $\begin{array}{l}\text { (((((right ventricle) OR (right ventricular)) } \\
\text { OR (right heart)) OR (cardiac function)) OR } \\
\text { (ventricular function)) OR (((echocardiographic) } \\
\text { OR (computed tomography)) OR (magnetic } \\
\text { resonance imaging)) }\end{array}$ \\
\hline \# 2 & (COVID-19) OR (SARS-CoV-2) \\
\hline \# 3 & \# 1 and \# 2 \\
\hline \multicolumn{2}{|l|}{ EMBase } \\
\hline \# 1 & $\begin{array}{l}\text { right ventricle OR right ventricular OR right heart } \\
\text { OR cardiac function OR ventricular function OR } \\
\text { echocardiographic OR computed tomography } \\
\text { OR magnetic resonance imaging }\end{array}$ \\
\hline \# 2 & 'COVID-19 19’ OR ‘sars cov 2’ \\
\hline \# 3 & \# 1 and \# 2 \\
\hline
\end{tabular}

ISI Knowledge via Web of Science

$\begin{array}{ll}\text { \#1 } & \text { TOPIC: (right ventricle) OR TOPIC: (right } \\ & \text { ventricular) OR TOPIC: (right heart) OR TOPIC: } \\ & \text { (cardiac function) OR TOPIC: (ventricular } \\ & \text { function) OR TOPIC: (echocardiographic) OR } \\ & \text { TOPIC: (computed tomography) OR TOPIC: } \\ & \text { (magnetic resonance imaging) } \\ & \text { Timespan: All years. Databases: WOS, BIOSIS, } \\ & \text { KJD, MEDLINE, RSCI, SCIELO. } \\ & \text { Search language=Auto } \\ & \text { TOPIC: (COVID-19) OR TOPIC: (SARS-CoV-2) } \\ & \text { Timespan: All years. Databases: WOS, BIOSIS, } \\ & \text { KJD, MEDLINE, RSCI, SCIELO. } \\ & \text { Search language=Auto } \\ & \text { \# } 1 \text { and \# } 2\end{array}$

\begin{tabular}{ll} 
MedRxiv & \\
\#1 & $\begin{array}{l}\text { ((((right ventricle) OR (right ventricular)) } \\
\text { OR (right heart)) OR (cardiac function)) OR } \\
\text { (ventricular function)) OR (((echocardiographic) } \\
\text { OR (computed tomography)) OR (magnetic } \\
\text { resonance imaging)) }\end{array}$ \\
\# 2 & (COVID-19) OR (SARS-CoV-2) \\
\# 3 & \# 1 and \# 2 \\
BioRxiv & \\
\# 1 & (((((right ventricle) OR (right ventricular)) \\
& $\begin{array}{l}\text { OR (right heart)) OR (cardiac function)) OR } \\
\text { (ventricular function)) OR (((echocardiographic) }\end{array}$ \\
& $\begin{array}{l}\text { OR (computed tomography)) OR (magnetic } \\
\text { resonance imaging)) }\end{array}$ \\
\# 2 & (COVID-19) OR (SARS-CoV-2) \\
\# 3 & \# 1 and \# 2 \\
\hline
\end{tabular}

COVID-19. No language limitation will be set for the inclusion of eligible studies. Studies reporting imaging RV involvement in other coronary virus infections (SARS 


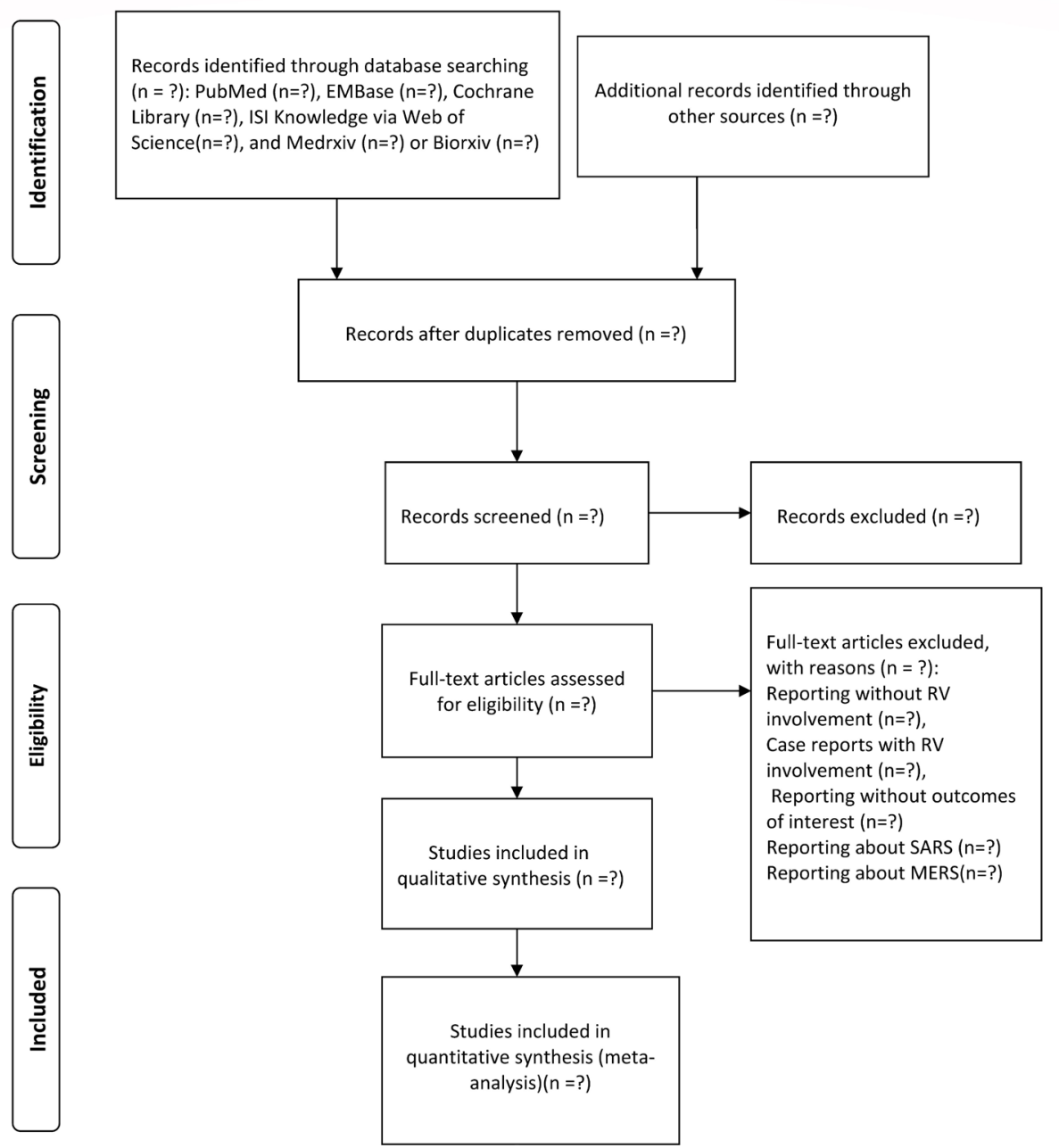

Figure 1 Searching process flowchart for eligible trials Selection. MERS, Middle East respiratory syndrome coronavirus; RV involvement, right ventricular involvement.

or Middle East respiratory syndrome coronavirus) will be excluded. Studies without reporting the outcomes of interest will also be excluded.

\section{Type of outcomes}

The primary outcome will be the incidence of RV involvement (dysfunction and/or dilation) assessed by echocardiography, CT or MRI. The definition criteria of RV dysfunction will be as follows: (1) fractional area change (FAC) $<35 \%$, tricuspid annular plane systolic excursion (TAPSE) $<17 \mathrm{~mm}$ and/or $\mathrm{S}^{\prime}<10 \mathrm{~mm} / \mathrm{seg}$; (2) 3D-(RV ejection fraction) RVEF $<45 \%$; (3) RV free wall longitudinal strain (RV-FWLS) $>-20 \%$. The definition criteria of RV dilation will be as follows: (1) RV to LV basal diameter classified as mild (0.67-0.9), moderate (1.0) and severe (>1.0) enlargement; (2) RV basal diameter $>41 \mathrm{~mm}$.

Secondary outcomes will include the risk factors for RV involvement the association with all-cause mortality during hospitalisation. Additional outcomes will be RV global longitudinal strain (RV-GLS) or RV-FWLS, TAPSE, S', FAC, RVEF, and RV diameter.

\section{Data extraction}

Data extraction will be completed by two independent authors (BL and HL). A third author (XW) will make the final decision in case of disagreements. Data extraction will consist of study design (author, publication year, country, retrospective or prospective type and sample size), patient characteristics (mean age, male proportion, diabetes proportion, hypertension proportion, hyperlipidaemia proportion, smoking proportion, coronary artery disease proportion, previous myocardial infarction, chronic heart failure, atrial fibrillation, previous peripheral vascular disease, previous stroke or transient ischaemic accident, acute or chronic kidney dysfunction, previous lung disease, pulmonary hypertension proportion, LV ejection fraction, elevated cTn proportion, elevated BNP proportion, acute LV failure proportion, beta-blocker usage, statin usage, ACE inhibitor/angiotensin receptor blocker usage, calcium channel blocker usage, aspirin usage, sacubitril/valsartan and diuretics), follow-up period, type of RV involvement, different categories of RV involvement and imaging methods (echocardiography, CT or MRI). 


\section{Risk of bias assessment}

The methodological quality evaluation of the studies will be evaluated by the Newcastle-Ottawa quality assessment scale. $^{20}$

\section{Data synthesis}

The incidence of imaging RV involvement including RV dysfunction and/or dilation in each study will be calculated. The OR or HR and 95\% CI in each study for the risk factors of RV involvement and association with all-cause mortality will be extracted. For continuous outcomes of RV-GLS, RV-FWLS, TAPSE, FAC and RV diameter (reported as the mean $\pm \mathrm{SD}$, median and $\mathrm{IQR}$, or median and range), we will calculate mean differences for each study and used weights (the inverse variance of the estimate) to pool the estimate (weighted mean difference) with 95\% CI. The DerSimonian and Laird random-effects model will be used in the pooled analysis for potential clinical inconsistency. Univariable or multivariable metaregression and subgroup analyses will be conducted for related risk factors (especially acute or chronic pulmonary embolism and pulmonary hypertension) and the association of RV involvement with all-cause mortality. Sensitivity analyses will be used to assess the robustness of our results by removing each included study at one time to obtain and evaluate the remaining overall estimates of $\mathrm{RV}$ involvement incidence and related risk factors, association with all-cause mortality, and other RV parameters (RV-GLS, RV-FWLS, TAPSE, FAC and RV diameter). If different severity categories are reported, we will choose the category without RV involvement as a reference. We will pool the ORs by combining all the categories of RV involvement for comparison with and without RV involvement categories using the DerSimonian and Laird random-effects model. If a study reports multiple categories ( $>2$ categories), we will calculate the OR using data on the number of cases and non-cases in all of the categories. Both linear and cubic spline regression models will be used to explore the dose-response relationship between different categories of RV involvement and the risk of mortality (OR or HR). ${ }^{21} 22$ Publication bias assessment will be performed with the Begg's and Egger's tests. $\mathrm{P}<0.05$ (two-sided) will be considered statistically significant. All statistical analyses will be performed in Stata software (V.10.0, StataCorp) and RevMan software (V.5.0, Cochrane Collaboration, Oxford, UK).

\section{DISCUSSION}

Although the primary target of COVID-19 is the respiratory system, the cardiovascular system could also be affected by the following major mechanisms: (1) COVID-19 directly binds to the renin-ACE2 receptor in the cardiovascular system, ${ }^{23}$ interferes with the related downstream signalling pathway, significantly affects the neurohumoral regulation of the cardiovascular system and directly causes myocardial injury. (2) COVID-19 binds to ACE2 receptors in the respiratory system, ${ }^{23}$ which directly causes lung injury and affects respiratory function. Hypoxia could directly affect the balance of myocardial oxygen supply and demand, or indirectly cause right heart damage by pulmonary hypertension. (3) In many critical cases, COVID-19 can cause acute systemic inflammatory reactions and cytokine storms, ${ }^{24}$ resulting in systemic organ dysfunction or failure to cause myocardial injury directly or indirectly. All of these factors could potentially affect the structure and function of the RV.

The imaging tools for RV structure and function assessment includes echocardiography, CT and cardiac Magnetic Resonance Imaging (CMR) in a direct way with more information than other indirect methods (such as electrocardiography, X-ray or central venous pressure), Although CT and CMR possess some unique advantages, 2D with 3D echocardiography could also provide non-invasive, convenience, fast and comprehensive RV assessment with low cost for subsequent therapeutic treatment guidance, ${ }^{25}$ In addition, point-of-care evaluation using echocardiography is quite meaningful for critical patients with COVID-19 infection in intensive care unit. Specifically, echocardiographic evaluation for patients with COVID-19 could reduce the time of transportation and thereby the cross-over contamination in hospital. In this study, TAPSE, FAC, RV-GLS, RV-FWLS and RV diameter will be chosen as the second outcomes. These echocardiographic parameters are regular and useful for RV structural and function assessment, and have been recommended in the recent guideline for patients with COVID-19. ${ }^{26-28}$

The major strength of this systematic review and metaanalysis will be the first comprehensive summary of the risk factors and the prognostic effect of imaging RV involvement in patients with COVID-19. Moreover, this meta-analysis will focus on the incidence of imaging RV involvement with different outcomes in patients with COVID-19. There are several limitations in our analysis. First, the majority of included study will be retrospective in design. Thus, inherit bias cannot be ruled out. Second, the sample size and the number of studies may be small due to the limited usage of imaging tools for cardiac assessment in patients with COVID19. Third, we could not rule out the potential influence of different definitions of RV dysfunction in COVID-19. Fourth, the impact of different severities of COVID-19 associated with RV involvement on adverse clinical outcomes needs further studies.

\section{ETHICS AND DISSEMINATION}

There is no need for ethical approval for the systematic review protocol according to the Institutional Review Board/Independent Ethics Committee of Fuwai Hospital. This meta-analysis will be disseminated through a peerreviewed journal for publication.

\section{Author affiliations}

${ }^{1}$ Department of Anesthesiology, State Key Laboratory of Cardiovascular Disease, Fuwai Hospital, National Center for Cardiovascular Diseases, Chinese Academy of Medical Sciences \& Peking Union Medical College, Beijing, China

${ }^{2}$ Department of Radiology, Beijing Hospital, National Center of Gerontology; Institute of Geriatric Medicine, Chinese Academy of Medical Sciences, Beijing, China 
${ }^{3}$ Department of Echocardiography, State Key Laboratory of Cardiovascular Disease, Fuwai Hospital, National Center for Cardiovascular Diseases, Chinese Academy of Medical Sciences \& Peking Union Medical College, Beijing, China

${ }^{4}$ Department of Cardiac Surgery, State Key Laboratory of Cardiovascular Disease, Fuwai Hospital, National Center for Cardiovascular Diseases, Chinese Academy of Medical Sciences \& Peking Union Medical College, Beijing, China

Contributors $\mathrm{CZ}$ and FD contributed to the conception and design of the study, and revision of the protocol. The manuscript of the protocol was drafted by BL. HL and XW will independently search and select the eligible studies and extract the data from the included studies. $\mathrm{HL}$ and $\mathrm{HA}$ will assess methodological quality and the risk of bias. All the authors approved the protocol publication.

Funding This work was supported by the Beijing Municipal Science \& Technology Commission (No. Z201100005520004), and National Natural Science Foundation of China (81771826 and 81970290).

Competing interests None declared.

Patient and public involvement Patients and/or the public were not involved in the design, or conduct, or reporting, or dissemination plans of this research.

Patient consent for publication Not required.

Provenance and peer review Not commissioned; externally peer reviewed.

Open access This is an open access article distributed in accordance with the Creative Commons Attribution Non Commercial (CC BY-NC 4.0) license, which permits others to distribute, remix, adapt, build upon this work non-commercially, and license their derivative works on different terms, provided the original work is properly cited, appropriate credit is given, any changes made indicated, and the use is non-commercial. See: http://creativecommons.org/licenses/by-nc/4.0/.

ORCID iD

Chenghui Zhou http://orcid.org/0000-0001-6428-6069

\section{REFERENCES}

1 Worldometersinfo. COVID-19 coronavirus outbreak 2020. Identification. Available: https://www.worldometers.info/coronavirus/ 2020

2 Grasselli G, Greco M, Zanella A, et al. Risk factors associated with mortality among patients with COVID-19 in intensive care units in Lombardy, Italy. JAMA Intern Med 2020;180:1345-55.

3 Zhao Z, Chen A, Hou W, et al. Prediction model and risk scores of ICU admission and mortality in COVID-19. PLoS One 2020;15:e0236618.

4 Armstrong RA, Kane AD, Cook TM. Outcomes from intensive care in patients with COVID-19: a systematic review and meta-analysis of observational studies. Anaesthesia 2020;75:1340-9.

5 Fried JA, Ramasubbu K, Bhatt R, et al. The variety of cardiovascular presentations of COVID-19. Circulation 2020;141:1930-6.

6 Zhou F, Yu T, Du R, et al. Clinical course and risk factors for mortality of adult inpatients with COVID-19 in Wuhan, China: a retrospective cohort study. Lancet 2020;395:1054-62.

7 Ashraf H, Rosenthal JL. Right heart failure: causes and clinical epidemiology. Cardiol Clin 2020;38:175-83.

8 Kholdani CA, Fares WH. Management of right heart failure in the intensive care unit. Clin Chest Med 2015;36:511-20.

9 Tan TC, Dudzinski DM, Hung J, et al. Peri-operative assessment of right heart function: role of echocardiography. Eur J Clin Invest 2015;45:755-66.
10 Song Y, Zhang M, Yin L, et al. COVID-19 treatment: close to a cure? $A$ rapid review of pharmacotherapies for the novel coronavirus (SARS-CoV-2). Int J Antimicrob Agents 2020;56:106080.

11 Bhavana V, Thakor P, Singh SB, et al. COVID-19: pathophysiology, treatment options, nanotechnology approaches, and research agenda to combating the SARS-CoV2 pandemic. Life Sci 2020;261:118336.

12 Mahmoud-Elsayed HM, Moody WE, Bradlow WM, et al. Echocardiographic findings in patients with COVID-19 pneumonia. Can J Cardiol 2020;36:1203-7.

13 Szekely Y, Lichter Y, Taieb P, et al. Spectrum of cardiac manifestations in COVID-19: a systematic echocardiographic study. Circulation 2020;142:342-53.

$14 \mathrm{Kim}$ J, Volodarskiy A, Sultana R, et al. Prognostic utility of right ventricular remodeling over conventional risk stratification in patients with COVID-19. J Am Coll Cardiol 2020;76:1965-77.

15 Bursi F, Santangelo G, Sansalone D, et al. Prognostic utility of quantitative offline 2D-echocardiography in hospitalized patients with COVID-19 disease. Echocardiography 2020;37:2029-39.

16 Dweck MR, Bularga A, Hahn RT, et al. Global evaluation of echocardiography in patients with COVID-19. Eur Heart J Cardiovasc Imaging 2020;21:949-58.

$17 \mathrm{Li} \mathrm{Y,} \mathrm{Li} \mathrm{H,} \mathrm{Zhu} \mathrm{S,} \mathrm{et} \mathrm{al.} \mathrm{Prognostic} \mathrm{value} \mathrm{of} \mathrm{right} \mathrm{ventricular}$ longitudinal strain in patients with COVID-19. JACC Cardiovasc Imaging 2020;13:2287-99.

18 Schott JP, Mertens AN, Bloomingdale R, et al. Transthoracic echocardiographic findings in patients admitted with SARS-CoV-2 infection. Echocardiography 2020;37:1551-6.

19 Shamseer L, Moher D, Clarke M, et al. Preferred reporting items for systematic review and meta-analysis protocols (PRISMA-P) 2015: elaboration and explanation. BMJ 2015;350:g7647.

20 Murad MH, Sultan S, Haffar S, et al. Methodological quality and synthesis of case series and case reports. BMJ Evid Based Med 2018;23:60-3.

21 Li Y, Pei H, Bulluck H, et al. Periprocedural elevated myocardial biomarkers and clinical outcomes following elective percutaneous coronary intervention: a comprehensive dose-response metaanalysis of 44,972 patients from 24 prospective studies. Eurolntervention 2020;15:1444-50.

22 Berlin JA, Longnecker MP, Greenland S. Meta-analysis of epidemiologic dose-response data. Epidemiology 1993;4:218-28.

23 Gheblawi M, Wang K, Viveiros A, et al. Angiotensin-converting enzyme 2: SARS-CoV-2 receptor and regulator of the reninangiotensin system: celebrating the 20th anniversary of the discovery of ACE2. Circ Res 2020;126:1456-74.

24 Pearce L, Davidson SM, Yellon DM. The cytokine storm of COVID-19: a spotlight on prevention and protection. Expert Opin Ther Targets 2020;24:723-30.

25 Surkova E, Muraru D, lliceto S, et al. The use of multimodality cardiovascular imaging to assess right ventricular size and function. Int J Cardiol 2016;214:54-69.

26 Cameli M, Pastore MC, Soliman Aboumarie H, et al. Usefulness of echocardiography to detect cardiac involvement in COVID-19 patients. Echocardiography 2020;37:1278-86.

27 Skulstad H, Cosyns B, Popescu BA, et al. COVID-19 pandemic and cardiac imaging: EACVI recommendations on precautions, indications, prioritization, and protection for patients and healthcare personnel. Eur Heart $J$ Cardiovasc Imaging 2020;21:592-8.

28 Agricola E, Beneduce A, Esposito A, et al. Heart and Lung Multimodality Imaging in COVID-19. JACC Cardiovasc Imaging 2020;13:1792-808 